\title{
Preparation and Modification of Natural Rubber Blends For Water Purification Treatment Usage
}

\author{
Wenfa Dong ${ }^{1}$, Ruogu Tang ${ }^{2}$ \\ 1. Ningbo Beishi Petrol Co.LTD. Ningbo, China. 315204 \\ 2. Institute of Ecology, College of Life Sciences, Zhejiang University. Hangzhou, China. 310058
}

\begin{abstract}
Compared to other synthetic rubbers, natural rubber (NR) has relatively good eco-friendly properties which provides itself the great potentials in environmentally restricted areas. In this study, the natural rubber that applied in water purification industry was blended with styrene-butadiene rubber (SBR) to overcome its inherent shortages. To understand the relationship between curing system/sulfur-accelerant ratio and mechanical properties of vulcanizates, NR and SBR were physically mixed with different formulations under two-roll mixing mill, and examined vulcanization characteristics of the compound. Then we prepared a series of NR/SBR vulcanizated rubbers via conventional vulcanization (CV), effective vulcanization (EV) and semi-effective vulcanization (SEV) respectively basing on each formulation and optimum curing time. We examined the mechanical properties of NR/SBR vulcanizates including tensile strength, tear strength, elongation at break, modulus, Shore A hardness and and relative volume abrasion. The results indicated that NR/SBR vulcanizates prepared in different systems differed in mechanical properties. Vulcanizates prepared via CV showed higher tensile and tear strength; vulcanizates prepared via EV had high modulus and hardness, and vulcanizates prepared via SEV performed high abrasion resistance. In the CV system, sulfur-accelerant ratio affected the mechanical properties. As the sulfur-accelerant ratio increased from 2.92 to 3.75 , the strength of tensile and tear, elongation at break of vulcanizates increased at first then decreased, while the modulus and Shore A hardness increased. The Relative volume abrasion decreased at first and then increased. In the EV system, sulfur-accelerant ratio also showed to affect the mechanical properties. As the sulfur-accelerant ratio increased from 0.2 to 0.42 , the tensile strength and tear strength increased, the elongation at break decreased, and the rebound resilience and Relative volume abrasion maintained at a range. The strength modulus and Shore A hardness changed irregularly. Compared to CV and EV, in SEV system that the sulfuraccelerant ratio was 1.14 , the abrasion resistance of vulcanizates was significantly improved, but the strength and hardness was improved slightly. The Relative volume abrasion reached $173 \mathrm{~mm}^{3}$. The other mechanical properties of vulcanizates from SEV system were between the CV and EV. The results from our study demonstrated that both vulcanization system and sulfur-accelerant ratio can affect the mechanical properties, and the $\mathrm{CV}$ can provide vulcanizates better mechanical properties. In addition, the NR/SBR vulcanizates provided a homogeneous phase, and the vulcanizates with different curing systems were stable in water environment up to 90 days. In addition, the biopolymeric chitosan derivatives were synthesized and physically introduced into the blends to provide water treatmentfunctions.
\end{abstract}

Key words: sulfur-accelerant ratio, stress concentration, crosslink bond, crosslink density 


\section{Introduction}

Natural rubber (NR polyisoprene), as mainly harvested from natural products, possesses relatively good ecofriendly properties compared to other synthetic rubbers ${ }^{[1]}$, therefore NR has been used in some environmentally restricted areas. To overcome its inherent shortages, NR has been widely blended with styrene-butadiene rubber (SBR). NR/SBR combines the advantages of NR and SBR and possess fine comprehensive qualities so that it could be practically used in some specific fields (e.g. water treatment industry) ${ }^{[1,2]}$. Currently, many NR/SBR vulcanizates are being used in dynamic environments, which require NR/SBR with fine mechanical properties in both static and dynamic state ${ }^{[2]}$. Thus, examinations and analysis of the comprehensive mechanical properties of NR/SBR are necessary before their industrial application.

Researches have demonstrated curing system directly affected on vulcanizates' mechanical properties besides constituents' structures and characteristics ${ }^{[3-13]}$. Curing system is always concerned because it can directly alerts vulcanizates' structures as well as affects vulcanizates' dynamical mechanical behaviors, and further determining mechanical properties ${ }^{[10-13]}$. In most cases, sulfur curing systems were used to prepare NR/SBR vulcanizates ${ }^{[2]}$. According to the constituents' ratio of curing systems, there are three kinds of curing systems: conventional vulcanization (CV), effective vulcanization (EV) and semi-effective vulcanization (SEV) ${ }^{[2]}$. The most significant difference among these three systems is the sulfur-accelerant ratio (it means the ration of the amount of sulfur and the amount of accelerant). The sulfur-accelerant ratio is usually higher than 2.5 in $\mathrm{CV}$, while the sulfur-accelerant ratio in EV is usually less than 0.4 and the sulfur-accelerant ratio is nearly equal to 1 in SEV. The difference in sulfur-accelerant ratio affects the type of crosslink bond and crosslink density of vulcanizates, consequently affects the vulcanizates' configuration, regularity, and crystallization or orientation behaviors, and will finally determine vulcanizates' mechanical properties. Thus, screening appropriate curing system is critical in a given environment. Some researches reported that $\mathrm{CV}$ system is suitable for vulcanizates in dynamic environment and EV system is good for that in static environment with high temperature ${ }^{[14-19]}$. However, these experiences didn't comparatively test the effects brought by curing systems and the sulfur-accelerant ratios on vulcanizates.

Therefore, in this study, the NR that used in water treatment application was selected for blending with SBR, and biopolymeric derivatives were introduced to provide bio-activities for water treatment usage. The NR/SBR was vulcanizated via conventional vulcanization (CV), effective vulcanization (EV) and semi-effective vulcanization (SEV) systems respectively. In the following test, we then examined the mechanical properties of the prepared NR/SBR vulcanizates. These mechanical properties include tensile strength, tear strength, elongation at break, modulus, Shore A hardness and relative volume abrasion.

\section{Experiment Section}

\subsection{Materials and instruments}

The compound formulation for preparing NR/SBR under the three curing systems was indicated in Table 1 . 
Table .1 Compound formulations for the three curing systems (unit: g)

\begin{tabular}{cccc}
\hline Compounds added & CV & EV & SEV \\
\hline NR & 180 & 180 & 180 \\
BR & 120 & 120 & 120 \\
HAF & 150 & 150 & 150 \\
Silica & 75 & 75 & 75 \\
Oil & 75 & 75 & 75 \\
ZnO & 15 & 15 & 15 \\
Stearic acid & 6 & 6 & 6 \\
Paraffin & 3 & 3 & 3 \\
CZ & 3 & 3 & 3 \\
$4010 \mathrm{NA}$ & 3 & 3 & 3 \\
TMTD & 0.6 & $2.7-4.5$ & 1.8 \\
Precipitated $\mathrm{CaCO}_{3}$ & 30 & 30 & 30 \\
$\mathrm{~S}$ & $10.5-13.5$ & $1.5-2.4$ & 4.2 \\
\hline
\end{tabular}

The devices used in our study were two roll rubber mixing mill (Shanghai Institute of Mechanical Technology), rotorless curemeters (GT-M2000-A, GOTECH CO LTD, Taiwan), Daylight press (Jiaxin Electric Device Technology CO LTD), electronic tensile testing machines, Shaore A durometer, rotating cylindrical drum device (GT-7012-D, GOTECH CO LTD, Taiwan) and densimeter (Preciso CO LTD).

\subsection{The preparation of NR/SBR vulcanizates}

We prepared the NR/SBR compound basing on ISO 2393:2008 and prepared NR/SBR vulcanizates basing on ISO 3417:1991. Examination of curing property was based on ISO 6502:1991. Temperature and pressure for preparing vulcanizing were $145^{\circ} \mathrm{C}$ and $10 \mathrm{MPa}$ respectively. Optimum curing time was obtained from the curing curve (Table 2). After preparation, the morphologies of NR/SBR vulcanizates were investigated by scanning electron microscopy (SEM). To prepared the corresponding biopolymeric products, the chitosan derivatives (include the schiff base and self-assembly particles) were used as bio-additives (with 5\% wt addition) in the blends (see supportive information).

\subsection{Examination of NR/SBR vulcanizates}

We measured tensile strength basing on ISO 37: 2005, and tear strength basing on ISO 34-1:1994, rebound resilience basing on ISO 4662-1986, modulus and elongation at break basing on ISO 37:2005, Shore A hardness basing on to ISO 7619-1:2004, relative volume abrasion basing on the ISO 4649: 2002 and density basing on the ISO 2781:2007. The water stability tests were conducted based on a previous report ${ }^{[6]}$.

Table 2 Curing time for each vulcanizates in given sulfur-accelerantratios

\begin{tabular}{ccc}
\hline Treatment No. & s/a & Optimum curing time \\
\hline 1 & 3.75 & $6^{\prime} 44^{\prime \prime}$ \\
\hline
\end{tabular}




\begin{tabular}{lcc}
\hline 2 & 3.33 & $7^{\prime} 41^{\prime \prime}$ \\
3 & 2.92 & $6^{\prime} 15^{\prime \prime}$ \\
4 & 1.14 & $5^{\prime} 06^{\prime \prime}$ \\
5 & 0.42 & $7^{\prime} 00^{\prime \prime}$ \\
6 & 0.36 & $9^{\prime} 55^{\prime \prime}$ \\
7 & 0.2 & $10^{\prime} 18^{\prime \prime}$ \\
\hline
\end{tabular}

\section{Results and Discussion}

The results from our study showed that vulcanizates prepared via CV had higher tensile and higher tear strength, while vulcanizates prepared by EV had high modulus and hardness, and vulcanizates prepared through SEV possessed high abrasion resistance (Table 3 and 4). In our study, the differences of mechanical properties were mainly caused by the differences of crosslink bonds and crosslink density among the three curing systems. The processing techniques also resulted into these differences.

Table 3 Experiment record of mechanical properties under CV

\begin{tabular}{llll}
\hline \multirow{2}{*}{\multicolumn{1}{c}{ Parameters tested }} & \multicolumn{3}{c}{ Vulcanizates } \\
\cline { 2 - 4 } & 1 & 2 & 3 \\
& $(\mathrm{~s} / \mathrm{a}=3.75)$ & $(\mathrm{s} / \mathrm{a}=3.33)$ & $(\mathrm{s} / \mathrm{a}=2.92)$ \\
\hline Tensile strength/MPa & 16.8 & 17.2 & 16.6 \\
Tear strength/kN*m ${ }^{-1}$ & 35.0 & 37.9 & 36.5 \\
Elongation at break/\% & 361 & 379 & 344 \\
Modulus (100\% elongation)/MPa & 3.5 & 3.3 & 3.3 \\
Modulus (200\% elongation)/MPa & 8.3 & 7.8 & 7.5 \\
Modulus (300\% elongation)/MPa & - & 11.2 & 11.4 \\
Rebound resilience/\% compression set & 60 & 60 & 61 \\
Shore A hardness & 64 & 67 & 62 \\
Relative volume abrasion $/ \mathrm{mm}^{3}$ & 202 & 207 & 213 \\
\hline
\end{tabular}

Table 4 Experiment record of mechanical properties under EV/SEV

\begin{tabular}{lllll}
\hline & \multicolumn{4}{c}{ Vulcanizates } \\
\cline { 2 - 5 } \multicolumn{1}{r}{ Parameters tested } & 4 & 5 & 6 & 7 \\
& $(\mathrm{~s} / \mathrm{a}=1.14)$ & $(\mathrm{s} / \mathrm{a}=0.42)$ & $(\mathrm{s} / \mathrm{a}=0.36)$ & $(\mathrm{s} / \mathrm{a}=0.2)$ \\
\hline Tensile strength $/ \mathrm{MPa}$ & 15.2 & 14.8 & 14.2 & 13.7 \\
Tear strength $/ \mathrm{kN}^{*} \mathrm{~m}$ & 35 & 33.7 & 32.5 & 30.9 \\
Elongation at break/\% & 380 & 358 & 387 & 423 \\
\hline
\end{tabular}




\begin{tabular}{lllll}
\hline Modulus (300\% elongation)/MPa & 14.2 & 13.7 & 12.7 & 12.8 \\
Rebound resilience/\% & 58 & 58.5 & 59 & 57 \\
Shaore hardness & 68 & 72 & 73 & 70 \\
Relative volume abrasion $/ \mathrm{mm}^{3}$ & 173 & 221 & 222 & 217 \\
\hline
\end{tabular}

\subsection{Crosslink bond type and its effects on vulcanizates' mechanical behaviors}

Stress-strain behaviors.In sulfur curing system there are three main types of crosslink bond:polysulfur bond (usually existing in CV), disulfide bond and monosulfur bond (both usually exist in EV/SEV and monosulfur bonds usually existing in SEV). Among the three types of bonds, the bond energy of polysulfur bond is relative lower ${ }^{[2]}$ (Table.5), resulting in a flexible bond. Thus, when stress concentration effect appears, flexible polysulfur bond can change shape and the chain gets enough time to orient and crystallize. This relative lower bond energy also makes polysulfur bonds easy to break down and rearranged. Therefore, the stress concentration effect can be weakened. Meanwhile, energy was absorbed so that dynamic loss was reduced during the rearrangement process. Disulfide bond and monosulfur bond, however, are usually short and rigid. These kinds of bonds tend to break down rapidly under stress and there is little time for the chain to orient and crystallize. Also, most disulfide bonds and monosulfur bonds are chemical inert. They cannot rearrange after broken, resulting in a lower integrated strength. Thus, vulcanizates with more polysulfur bonds have better strength in general.

Table 5 Common crosslink bond type and bond energy

\begin{tabular}{lll}
\hline Crosslink bond type & Curing systems & Bond energy/kJ.mol ${ }^{-1}$ \\
\hline Monosulfur bond & EV/SEV & 284.7 \\
Disulfide bond & EV/SEV & 267.9 \\
Polysulfur bond & $\mathrm{CV}$ & $<265$ \\
\hline
\end{tabular}

Stress relaxation behaviors. The modulus and hardness usually were affected by stress relaxation behaviors. In our experiment, modulus and hardness were higher under EV (Table 3-4) that was induced by difference rates of stress relaxation in each vulcanizate. Here we used Parallel Maxwell Model ${ }^{[20]}$ to explain why there are differences in stress relaxation behaviors among the three curing system. The stress relaxation formula was as follow ${ }^{[21]}$

$$
\sigma=\sum_{i} \sigma_{0 i} e^{\frac{-t}{\tau_{i}}} \quad E=\sum_{i} \frac{\sigma_{0 i}}{\varepsilon_{0}} e^{\frac{-t}{\tau_{i}}}
$$

In these formulae, $\sigma_{0 \mathrm{i}}$ represents each Maxwell unit's stress, $\tau_{\mathrm{i}}$ represents each unit's relaxation time and $\mathrm{E}$ represents the Young's modulus. As mentioned above, polysulfur bonds are easier to change shape and rearrange. Therefore, flexible polysulfur bonds make vulcanizates' stress relaxation rate in CV system faster than that in EV and SEV. This means in CV system, $\tau_{i}$ is generally short and the modulus (hardness) is low. By 
contrast, disulfide bonds and monosulfur bonds can slow down the vulcanizates' stress relaxation rate and prolong the $\tau_{i}$ so that modulus and hardness were higher under EV.

Dynamic abrasion behaviors. Vulcanizates' abrasion behaviors include wear abrasion, fatigue abrasion and curl abrasion. All of those are related to the mechano-chemical active chain-oxydized reaction initiated by stress ${ }^{[22]}$. During the reaction, the chain and crosslink bonds will be broken down and form ether bonds ${ }^{[23]}$. Jia reported that the degree of abrasion is in direct proportion to the amount of crosslink bonds broken down in the vulcanizates ${ }^{[23]}$. Table 5 showed that among three types of bonds, monosulfur bond possesses highest bond energy so that it is not easy to be broken down in stress. Based on this, we suggested that in the same condition, vulcanizates prepared in SEV possess better abrasion resistance properties.

\subsection{Crosslink density and its effects on vulcanizates' mechanical properties}

From the Table 3 and Table 4, we found that the sulfur-accelerant ratio was influential on mechanical properties in each curing system. In the CV system, as the sulfur-accelerant ratio increased, the strength of tensile and tear, elongation at break of vulcanizates increased at first, reached the maximum and then decreased, while the modulus and Shore A hardness increased (except in 300\% elongation). The Relative volume abrasion decreased at first and then increased. In the EV system, as the sulfur-accelerant ratio, the tensile strength and tear strength increased, the elongation at break decreased, and the rebound resilience and Relative volume abrasion maintained at a range. The strength modulus and Shore A hardness changed irregularly. Compared to that in $\mathrm{CV}$ and $\mathrm{EV}$, product from SEV system possessed intermediate mechanical properties of vulcanizate.

Different sulfur-accelerant ratio impacts various crosslink densities. In sulfur curing system, crosslink density can affect vulcaniates' behaviors in both static and dynamic state. With the crosslink density increasing, vulcanizates may become three-dimensional and chain segments may have to overcome more constraints to move. Usually, the modulus, hardness, strength and elongation increase with the crosslink density increasing, However, we found that when crosslink density reached a certain value the modulus, hardness, strength and elongation did not increase with the crosslink density increasing. This phenomenon can be explained by NR's crystallize and orientate behaviors.

Crystallize behaviors. The Avrami formula often explains crystallize behaviors ${ }^{[21]}$. The Avrami formula indicates as follow.

$$
X_{c, t}^{V}=X_{c, \infty}^{V}\left(1-e^{-k t^{n}}\right)
$$

$X$ represented the degree of crystallinity of NR. $k$ is the crystallization rate constant. $t$ is the crystallization and $n$ is Avrami index. In our systems, we did not add any crystallization agents. Therefore, we could assume that the crystal nucleus formed homogeneously, and the main crystal structures were oblique crystal. The $n$ value thus can be a constant as 4 in this system ${ }^{[21]}$. Under this circumstance, only the $t$ and $k$ determines the degree of crystallinity. When crosslink density is beyond a certain range, the space for crystallization is 
replaced by extra crosslink bonds and $k$ will become smaller. And the time for NR to get crystallized under stress will be longer. Then the degree of crystallinity will be reduced, which further reduces strength.

Orientation behaviors: The orientation factor $\left(\mathrm{F}=\left(3 \cos ^{2} \theta-1\right) / 2\right)$ represents degree of the orientation ${ }^{[21]}$, the $\theta$ is angle between direction of rubber's chain and direction of orientation. Usually in NR or SBR, the chains orient partially random. However, chains will orient in the same direct with stress, which means the $\theta$ will reach 0 (the Fwill reach 1). If crosslink density is too concentrated, orientation direction will be seriously interfered, the $\theta$ will be changed and F will become smaller than expected. Thus, the vulcanizates' strength can be weakened.

\subsection{Vulcanization process techniques}

Vulcanization process techniques including especially temperature and curing time often affect crosslink bonds and crosslink density. The curing time were obtained from the curing curve (Table 2). Considering the environment difference between laboratory and factory, we prolonged 5 extra minutes to optimum curing time. Usually the formation of crosslink bonds are dependent on the curing time, but if the vulcanizate is heated too long, crosslink bond will break down and rearrange, the chain also reacts with each other ${ }^{[23]}$. Thus, the vulcanizate's appearance and qualities can be seriously affected. So, in our experiment, we set the time based on the theoretical curing time rather than optimum curing time.

Traditionally the temperature is set in the range from $143{ }^{\circ} \mathrm{C}$ (for NR) to $150{ }^{\circ} \mathrm{C}$ (for SBR) $)^{[1,2]}$. In this work, we controlled the temperature at $145{ }^{\circ} \mathrm{C} \pm 0.5{ }^{\circ} \mathrm{C}$. According to Van't Hoff formula ${ }^{[2]}$ and Arrhenius formula ${ }^{[2]}$, improving heating degree will shorten the curing time, but high temperature could lead vulcanizates chain breakdown and affect strength ${ }^{[24]}$. So we did not set temperature over $150{ }^{\circ} \mathrm{C}$ (Table 2).

\subsection{Morphologies characterization}

As shown in figure 1, the SEM images displayed that the NR/SBR vulcanizates, despite prepared by different curing systems, possessed a homogeneous phase, which means the NR and SBR are compatible.

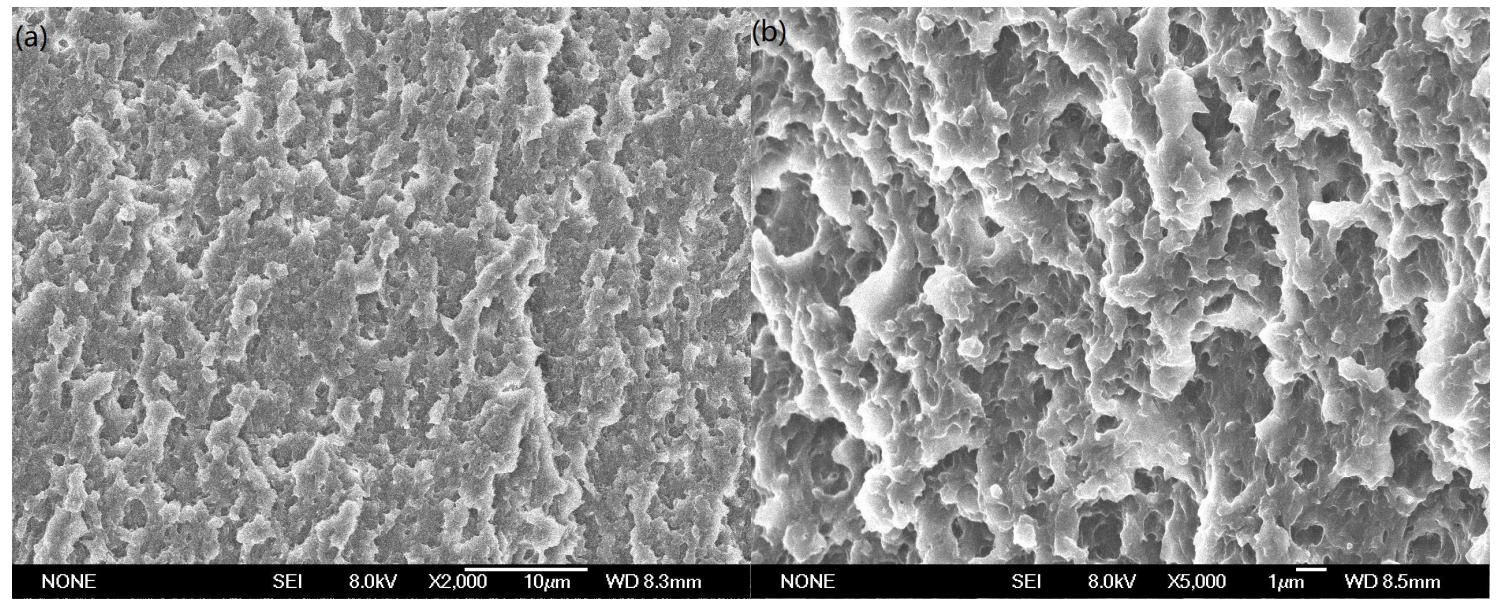




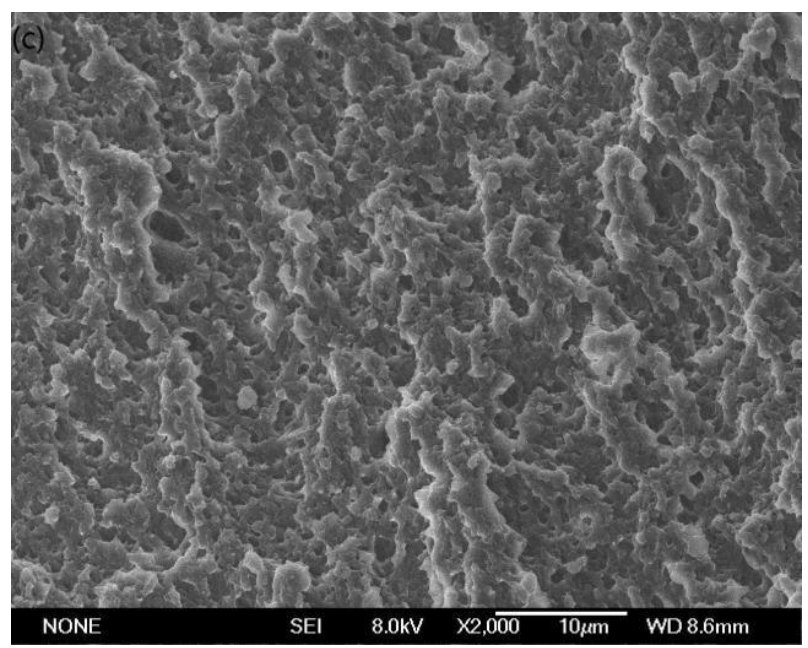

Fig 1. SEM images of NR/SBR vulcanizates prepared by CV (a), EV (b) and SEV (c) curing system.

\subsection{Water stability test}

Fig. 2 showed the water stabilities of NR/SBR vancanizates. After 90 dayes of treatment the mass increment could reach up to $3.8 \%$ and the increment became ceased to would not continue. The results indicated that the $\mathrm{NR} / \mathrm{SBR}$ vulcanizates were stable in aqueous environment and capable in water treatment industry.

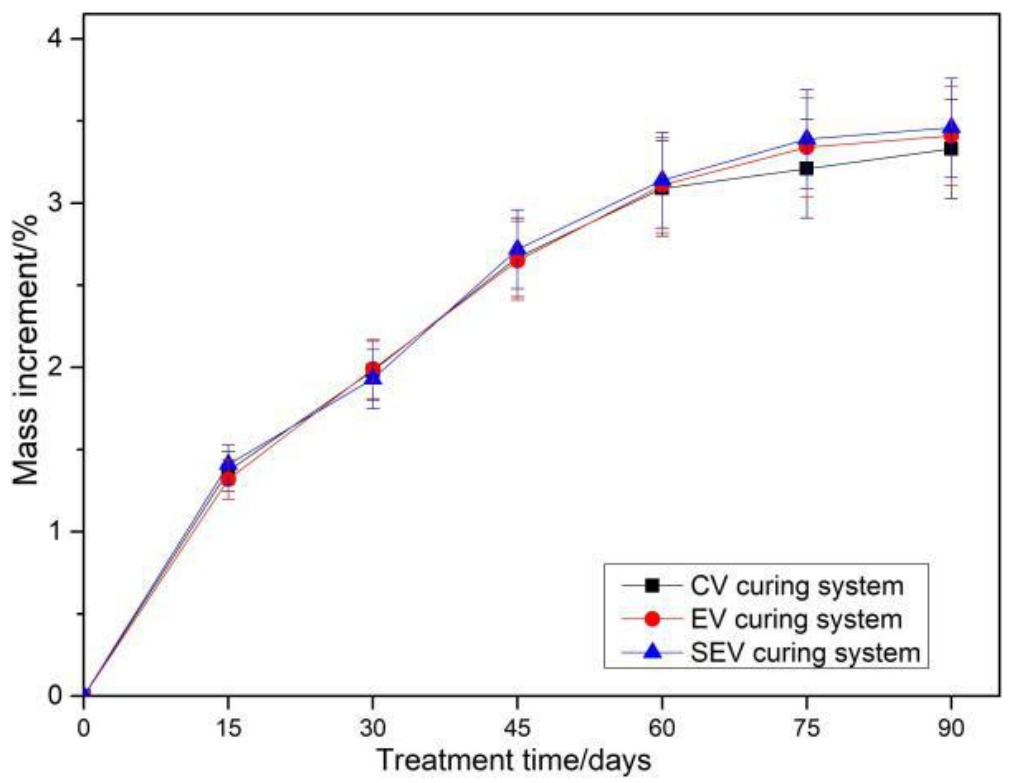

Fig.2 Water stability of NR/SBR vulcanizates

\subsection{Incorporation of biopolymeric derivatives}

The presence of chitosan in the derivatives could be confirmed by FT-IR subtraction spectra (shown in Fig.3), the peaks around $2900 \mathrm{~cm}^{-1}$ could be attributed to the alkyl groups from modified chitosan, peaks around $1050 \mathrm{~cm}^{-1}$ were related to the chitosan's cellulosic ring. The X-ray photoelectron spectroscopy (XPS) assessment also indicated the incorporation of chitosan derivatives as the nitrogen content (from the amino groups on chitosan) increased. 


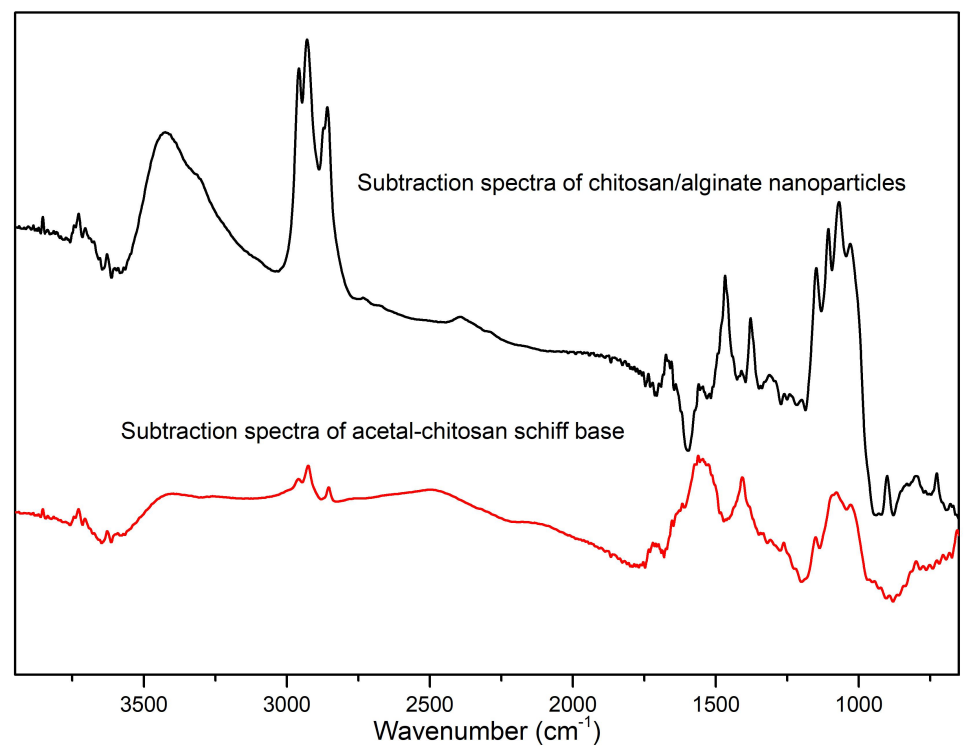

Fig.3 FT-IR subtraction spectra of chitosan incorporated NR/SBR blends (via SEV curing system)

Table 6 XPS test of nitrogen contents of NR/SBR blends (via SEV curing system)

\begin{tabular}{cc}
\hline & Nitrogen content (\%wt) \\
\hline NR/SBR & 0.03 \\
Acetal-chitosan schiff base incorporated NR/SBR & 0.44 \\
Chitosan/alginate incorporated NR/SBR & 0.31 \\
\hline
\end{tabular}

\section{Conclusion}

Curing system and the sulfur-accelerant ratio affected the mechanical properties of NR/SBR vulcanizates interactively. In our cases, CV can provide vulcanizates relative better strength, EV can provide vulcanizates relative better modulus and hardness, and SEV can provide better abrasion resistance. Our results indicated that mechanical properties of vulcanizates'varied non-linearly with the sulfur-accelerant ratio. There was an optimum value of sulfur-accelerant ratio for each mechanical property under the three curing systems. For CV system, optimum value of sulfur-accelerant ratio was 3.33, while for SEV/EV system, this ratio was 1.14 . The results improve our understanding to the effects of sulfur-accelerant ratio on mechanical properties in different curing systems.

\section{Acknowledgment}

The work was partially supported by the Natural Science Foundation of China. 


\section{References}

[1] Brydson J A. Rubber Materials and Their Compounds. London: Elsevier Applied Science. 1988.

[2] James E. Mark, Burak Erman, Frederick Roland Eirich. Science and Technology of Rubber. New York: Academic Press Inc. 2005.

[3] Soares B G, de Oliveira M, Meireles D, Sirqueira A S, Mauler R S.Dynamically vulcanized polypropylene/nitrile rubber blends: The effect of peroxide/bis-maleimide curing system and different compatibilizing systems. Journal of Applied Polymer Science. 2008, 110(6): 3566-3573.

[4] Zhang Xinxing, Lu Canhui, Mei Liang. Properties of natural rubber vulcanizates containing mechanochemically devulcanized ground tire rubber. Journal of Polymer Research. 2009, 16(4):411-419.

[5] Trifkovic M, Sheikhzadeh M, Choo K, Rohani S. Experimental and statistical study of the effects of material properties, curing agents, and process variables on the production of thermoplastic vulcanizates. Journal of Applied Polymer Science. 2010, 118(2): 764-777.

[6] Thitithammawong A, Noordermeer J W M, Kaesaman A, Nakason C. Influence of compatibilizers on the rheological, mechanical, and morphological properties of epoxidized natural rubber/polypropylene thermoplasticvulcanizates. Journal of Applied Polymer Science. 2008, 107(4): 2436-2443.

[7] Su Jun, Chen Shuangjun, Zhang Jun, Xu Zhongzi. Comparison of cure, mechanical, electric properties of EPDM filled with $\mathrm{Sm}_{2} \mathrm{O}_{3}$ treated by different coupling agents. Polymer Testing. 2009, 28(3):235-242.

[8] Tsai Yuhsin, Tsai Pingyuan, Wu Yaotsu, Leu Mingtsong, Wu Jyhhorng. Effect of Complex curatives on the properties for ethylene propylene diene rubber/polypropylene blends. Journal of Elastomers and Plastics. 2009, 41(3): 209-221.

[9] Mostafa A, Abouel-Kasem A,, Bayoumi M R, El-Sebaie MG. On the influence of CB loading on the creep and relaxation behavior of SBR and NBR rubber vulcanizates. Materials \& Design. 2009, 30(7): 2721-2725.

[10] Utara S, Boochathum P. Novel dynamic vulcanization of polyethylene and ozonolysed natural rubber blends: Effect of curing system and blending ratio. Journal of Applied Polymer Science. 2011, 120(5):2606-2614.

[11] Bu Jinglong, Jiang Zhengyi, Jiao Sihai. A comparative study of the effect of crosslink agents and chemical modification on properties of natural rubber vulcanizates. Advanced Materials Research. 2012, 415 - 417: 2334-2337.

[12] El-Nemr K F. Effect of different curing systems on the mechanical and physico-chemical properties of acrylonitrile butadiene rubber vulcanizates. Materials \& Design. 2011, 32(6): 3361-3369.

[13] Leite P R S, Soares B G, Sirqueira A S. Dynamically vulcanized polypropylene/styrene-butadiene rubber blends: The effect of a peroxide/bismaleimide curing system and composition. Journal of Applied Polymer Science. 2011, 120(2): 981-990.

[14] He Qiang, Jia Qibo, Lu Yonglai, Zhang Liqun. The relationship between crosslinked structure and physical properties of silicone rubber vulcanizates. China Rubber Industry. 2010, 57(5): 268-274.

[15] Sadhu S, Bhowmick A K. Preparation and properties of styrene-butadiene rubber based nanocomposites: the influence of the structural and processing parameters. Journal of Applied Polymer Science. 2004, 92(2):698-709.

[16] Zeng Zongqiang, Huang Maofang, Zhang Beilong, Liang Yue. Effect of preparation processes on properties of NR. China Rubber Industry. 2011, 58(7): 422-429.

[17] Mu Shouyong, Jiang Ping, Sun Xuehong, Zhao Shugao, Zhang Ping. Influence of different curing systems on physical properties of SSBR T2000R. China Elastomerics.

[18] Chen Peizhi, Jia Hongbing, Li Lin, Quan Jie, Wang Shizhao. Properties of high-vinyl SSBR with different curing systems. Journal of Nanjing University of Science and Technology (Natural Science). 2006,30(6): 774-777.

[19] Wang Yong, Zhou Qi, Gao Xinwen and Xin Zhenxiang. Influence of curing system on properties of NBR compound. China Rubbber Indusrty. 2008, 55(1): 28-30.

[20] Qiu Yiming, Wu Qiye, Zhou Jizhi, Jiao Mingqi. Simulation of stress relaxation curves of nature rubber. Polymer Materials Science and Technology. 1993, (4): 114-118.

[21] Wu Qiye, Zhang Ping, Yang Wenjun, Lin Runxiong. Polymer Physics. Beijing: High Education Press.2010.

[22] Jia Hongbing, Ji Qing-min, Jin Zhigang, Wen Wei, Zhang Shiqi, Wang Meixun. Study on abrasion of SBR vulcanizates with different structures. China Rubber Industry. 2000, 47(7): 387-391.

[23] Zhou Hongbin. Reversion kinetics of NR and main anti recovery agents. Tire Industry. 2000, 20(4): 195-198. 


\section{Supporting information}

Synthesis of chitosan based schiff base: $1 \mathrm{~g}$ chitosan was dissolved into $100 \mathrm{~mL} 1 \%(\mathrm{v} / \mathrm{v})$ acetic acid (in the round bottom flask), after that $10 \mathrm{~mL}$ acetaldehyde was added into the chitosan solution. The reaction was performed under $70^{\circ} \mathrm{C}$, regular pressure for 1 hour. After cooling down to the room temperature, $10 \%(\mathrm{w} / \mathrm{v})$ sodium hydroxide solution was added to adjust the $\mathrm{pH}$ to 10 . The precipitants were filtered and washed by ethanol and distill water until the $\mathrm{pH}$ reached at 7 . Finally, the products were dried under $40^{\circ} \mathrm{C}$.

Syntheiss of chitosan/alginate nanoparticles: $10 \mathrm{mg} / \mathrm{mL}$ alginate and chitosan solution were prepared at first. The chitosan solution was directly added into alginate solution with constant stirring $(180 \mathrm{rpm})$ for 120 minutes at $4^{\circ} \mathrm{C}$. After reaction, the solution was centrifuged at $3000 \mathrm{rpm}$ for 10 minutes. The upper layer solution and filtered by $0.45 \mu \mathrm{m}$ syringe filter, then the filtered solution was under dialysis for 24 hours to remove unreacted chitosan and alginate. The dialyzed solution was frozen in liquid nitrogen and then lyophilized for storage. 